Research Paper

\title{
AHNAK as a Prognosis Factor Suppresses the Tumor Progression in Glioma
}

\author{
Zijin Zhao ${ }^{1,2 *}$, Songhua Xiao ${ }^{3 *}$, Xianrui Yuan ${ }^{1,2}$, Jian Yuan ${ }^{1,2}$, Chi Zhang1, 2, Haoyu Li ${ }^{1,2}$, Jun Su1, Xiangyu \\ Wang1, Qing Liu1, ${ }^{1 凶}$ \\ 1. Department of Neurosurgery, Xiangya Hospital, Central South University, Changsha, Hunan, China; \\ 2. Institute of Skull Base Surgery and Neuro-oncology at Hunan, Changsha, China; \\ 3. Department of Neurology, Sun Yat-Sen Memorial Hospital, Sun Yat-Sen University, Guanzhou, Guangdong, China. \\ * These authors contributed equally to this work \\ $\square$ Corresponding author: Qing Liu, M.D. \& Ph.D., Professor and Vice Chairman, Department of Neurosurgery, Xiangya Hospital, Central South University, \\ Institute of Skull Base Surgery \& Neuro-oncology at Hunan, 87 Xiangya Road, Changsha, Hunan, 410008, P.R. China E-mail: liuqingdr@csu.edu.cn Tel: \\ +8615116215296; +8613657439109 Fax: +8673184327401 \\ (c) Ivyspring International Publisher. This is an open access article distributed under the terms of the Creative Commons Attribution (CC BY-NC) license \\ (https:// creativecommons.org/licenses/by-nc/4.0/). See http://ivyspring.com/terms for full terms and conditions.
}

Received: 2017.03.27; Accepted: 2017.07.07; Published: 2017.08.25

\begin{abstract}
Purpose: AHNAK is originally identified as a giant protein based on the estimated size of approximately $700 \mathrm{kDa}$. The aim of this study is to identify the role of AHNAK in the pathogenesis of glioma.

Methods: We tested AHNAK mRNA level in a panel of six human glioma cell lines; and in 30 cases of normal brain tissues and 73 cases of glioma tissue samples using a qRT-PCR method. Further, we analyzed the relationship of AHNAK expression with clinicopathological characteristics in glioma patients. Meanwhile, we analyzed the relationship of expression of AHNAK and survival of glioma patients in survival analyses. Then, in vitro, we analyzed the biological effects of AHNAK in glioma cell lines (U87 and U251) including proliferation assay, cell transwell assay, and apoptosis. And in vivo, we examined the effects of AHNAK on tumor growth using xenograft model of human glioma cells in nude mice. Then we examined the expression of $\mathrm{Ki}-67$-positive cells in these tumors.

Results: We found that the mRNA levels of AHNAK were down-regulated in 4 of 6 human glioma cell lines, especially in U87 and U251 cell lines. Meanwhile, in glioma patients, a negative correlation was found between the expression of AHNAK and the glioma histopathology. And a low expression of AHNAK was a significant and independent prognostic factor for poor survival of glioma patients. Through over expression of AHNAK in both of U87 and U251, we demonstrated that overexpression of AHNAK could inhibit glioma cell proliferation and invasion, induce apoptosis, and inhibit in vivo glioma tumor growth and ki-67 expression.

Conclusions: The AHNAK acts as a potential tumor suppressor. Our study provides a preclinical basis for developing AHNAK as a reliable clinical prognostic indicator for glioma patients, and a new biomarker for treatment response, and a potentially therapeutic target in glioma management options.
\end{abstract}

Key words: AHNAK; glioma; tumor suppression; prognosis; Ki-67.

\section{Introduction}

Glioma is one of the most common primary brain tumors with poor prognosis because of their highly invasive growth pattern and their frequent resistance to therapies [1, 2]. Within each histological subtype, they could be further categorized into grades I-IV lesions based on the degree of malignancy [3]. The clinical outcomes of glioma patients depend heavily on histopathological features [4]. Despite the development of multimodal and aggressive treatments that include surgical resection, local radiotherapy and systemic chemotherapy in the past decades, patient outcomes remain unsatisfactory [5, 6]. The characteristic progressive overgrowth and diffuse invasion may contribute to the overall poor prognosis of glioma [7]. Although growing numbers of studies have been focused on exploring the 
molecular modulatory network involved in the development of glioma and investigating the effective therapeutic targets [8], it is vital to identify new potential biomarker for early diagnosis, more accurate prognosis prediction, and novel therapeutic target [7].

AHNAK, or nucleoprotein AHNAK, meaning 'giant' in Hebrew, is originally identified as a large $700 \mathrm{kDa}$ protein that is differentially expressed in some cancer cell lines [9]. AHNAK is identified as a previously described protein, desmoyokin, a desmosomal plaque protein found in bovine muzzle epithelium [10]. The expression of AHNAK is subsequently localized to the plasma membrane of keratinocytes in human epidermis [11]. AHNAK has been reported in many intracellular locations including the nucleus, cytoplasm and plasma membrane [12-14]. Although AHNAK has been suggested for the presence of both a nuclear export signal and a nuclear localization signal, its intracellular trafficking seems to be dependent on cell type, the formation of cell-cell contacts, extracellular calcium concentrations and phosphorylation status $[12,13,15]$. The implicated pathways or cellular processes seem distinct with a role ranging from in the formation of the blood-brain barrier, in cell architecture and migration, in pluripotent stem cell generation, to the regulation of cardiac calcium channels and muscle membrane repair [12, 16-20]. As research continues, there is an increasing interest in the function of AHNAK in various malignancy tumors. There are obvious differences in AHNAK expression in different cancers. Concerning the present findings, the expression of AHNAK is down-regulated in human breast cancer [12], melanoma [21], Burkitt lymphoma, colon carcinoma, and neuroblastoma [9]; but up-regulated in mesothelioma [22], laryngeal carcinoma [23] and invasive ductal carcinoma [24]. Meanwhile, researches showed that AHNAK mutation was considered as a prognostic factor related to the poor survival of patients with melanoma [21], laryngeal carcinoma [23], triple-negative breast cancer [25] and glioblastoma [26].

However, the expression of AHNAK in glioma and its function has not been explored detailedly and deeply. Therefore, in order to identify the role of AHNAK in the pathogenesis of glioma, we analyzed the relationship of the expression of AHNAK in glioma cell lines and patients' tissues. The clinicopathological features of AHNAK including the survival of patients were assessed. In addition, the function of AHNAK in glioma was evaluated in mesothelioma cell lines, xenografts, and human specimens.

In this study, we found that AHNAK could affect glioma biological characteristics, which was lowly expressed in glioma tissues and cell lines, which might act as a tumor suppressor gene to suppress malignancy of glioma cells, including proliferation, invasion, and to promote cell apoptosis. Moreover, the relatively lower expression of AHNAK was significantly associated with malignant status and poor prognosis of glioma patients. Thus, we clarified the clinical significance and function of AHNAK in glioma by analyzing clinical and molecular pathology features in vitro and in vivo experiments.

\section{Materials and Methods}

\section{Cell culture and transfection}

The cell lines including human glioma cell lines (SF767, SF126, A172, SHG-44, U87 and U251) and normal gliocyte line (HEB) were obtained from the American Type Culture Collection (Manassas, VA, USA) and were passaged for less than six months after thawing frozen aliquots in the laboratory. All of these cells were maintained according to the supplier's instructions. The cell lines were authenticated by short-tandem repeat DNA profiling and were identified to be free of mycoplasma infection before use.

Lentivirus mediated AHNAK-expressing vector (EX-V0190-Lv122) and control vector were purchased from GeneCopoeia (Rockville, MD, USA). The cDNA of AHNAK plasmids were transfected into 293T cells $\left(1 \times 10^{6}\right)$ for $48 \mathrm{~h}$ to generate a lentivirus by using Lipofectamine ${ }^{\circledR} 2000$ (Invitrogen Life Technologies, Carlsbad, CA, USA) according to the manufacturer instructions. Control group was the vector-transfected group (EX-NEG-Lv122). The viral supernatant was subsequently collected and used to infect the U87 and U251. After $72 \mathrm{~h}$ post-transfection, western blotting was performed to determine the efficiency of transfection.

\section{Clinical samples and tissue specimens}

We obtained 73 cases of glioma data. The clinical data, including gender, age, follow-up, and outcome, were obtained from the medical records of Xiangya Hospital of Central South University, Hunan, China from March 2008 to November 2010. The cases in the study included 36 female and 37 male with the age ranging from 21 to 67 years, with a mean of 45 years (Table 1). The main clinical symptom included headache $(38 / 73,52.1 \%)$, epilepsy $(20 / 73,27.4 \%)$, hypomnesis $(7 / 73,9.6 \%)$, emesis $(5 / 73,6.8 \%)$ and other symptoms $(3 / 73,4.1 \%)$. The follow-up time was ranging from 3 months to 41.5 months, with a mean of 21.2 months. During the follow-up, 35 patients $(47.9 \%)$ were still alive and unfortunately, 38 patients $(52.1 \%)$ were died. We obtained frozen tissue samples 
of these 73 cases of glioma and corresponding other 30 cases of normal brain tissues from the same hospital. The Ethical Committee of the Faculty of Medicine, the Central South University approved this study, and all patients obtained the informed consent. Tumor samples were diagnosed by two pathologists blinded to patient data with the World Health Organization (WHO) system. In these glioma tissues, the glioma histopathology included low grade glioma (Grade I-II) 25 cases $(34.2 \%)$ and high grade glioma (Grade III-IV) 48 cases (65.8\%).

Table 1. Analysis of the correlation between expression of AHNAK in primary glioma and its clinicopathological parameters

\begin{tabular}{lllll}
\hline Viable & Cases & \multicolumn{3}{l}{ AHNAK } \\
\cline { 3 - 5 } & & low & high & P value \\
\hline Age (years) & & & & \\
$\leq 45$ & 31 & 24 & 18 & 0.348 \\
$>45$ & & & 11 & \\
Gender & 36 & 21 & 15 & 0.462 \\
Male & 37 & 23 & 14 & \\
Female & & & & \\
Glioma histopatholgy & 25 & 8 & 17 & $<0.001$ \\
Grade I-II & 48 & 36 & 12 & \\
Grade III-IV & & & & \\
\hline
\end{tabular}

\section{Quantitative RT-PCR analysis (qRT-PCR)}

The total RNA from the cells was extracted with Trizol reagent (Invitrogen, Carlsbad, CA, USA). The concentrations of the RNA samples were determined by using NanoDrop ND-1000 instrument. The electrophoresis on a denaturing agarose gel assessed the integrity of RNA. The QuantiFast SYBR® Green RT-PCR Kit (QIAGEN, Germantown, MD, USA) which is a one-step RT-PCR kit was used to perform the reverse transcription and qRT-PCR reactions. Each reaction was repeated in triplicates. The fold change was determined as 2-ddCt, where $\mathrm{Ct}$ is the fractional cycle number at which the fluorescence of each sample passes the fixed threshold. The Bio-Rad IQTM5 Multicolour Real-Time PCR Detection System (USA) was used to perform all of the real-time PCR assays. The expression levels of the genes were normalized to the housekeeping gene $\beta$-actin as a control. The average expression of AHNAK mRNA was taken as the dividing point meaning that high expression was higher than the average expression and low expression was lower than average expression.

\section{Cell proliferation assay}

Cell suspension was plated on 6-well plates with the concentration of $3 \times 10^{4} / \mathrm{ml}$. The numbers of cells were counted using a Coulter Counter (Beckman Coulter, Fullerton, USA) after the incubation of 1, 2, 3 and 4 days respectively in triplicate.

\section{Cell invasion assay}

Cells in serum-free medium were seeded into the upper chamber with Matrigel in the insert of a 24-well culture plate (BD Biosciences, MA) and 15\% fetal bovine serum was added to the lower chamber as a chemoattractant. After 48 hours of incubation, Invasive cells adhering to the lower membrane of the inserts were stained with Crystal Violet, counted and imaged.

\section{MTT assay}

U87 and U251 cells transfected with control plasmids or AHNAK-expressing vector were seeded in 96-well plate for $12 \mathrm{hr}$. After that, $100 \mu \mathrm{l}$ of DMEM containing $5 \mathrm{mg} / \mathrm{ml}$ MTT were added into the wells, and incubated $37^{\circ} \mathrm{C}$ for $4 \mathrm{~h}$. The MTT medium was removed, and $50 \mu \mathrm{l}$ of DMSO was added, and incubated at $37^{\circ} \mathrm{C}$ for $15 \mathrm{~min}$. The optical density (OD) at $570 \mathrm{~nm}$ was measured using the ELx800 Absorbance Microplate Reader (Biotek, USA). The experiments were repeated three times. The inhibition rate was calculated by using the equation: (OD of scramble group - OD of AHNAK group)/ OD of scramble group $\times 100 \%$.

\section{Immunohistochemical Staining}

Tumor tissues were cut into $4 \mu \mathrm{m}$ thick sections and transferred to positively charged slides. Then, the slides were deal with dewaxing, rehydration, blocking with hydrogen peroxide, and antigen retrieval with microwave (Dako target retrieval solution, citrate buffer with $\mathrm{pH}$ 6.0). After that, the slides were incubated at $2-8{ }^{\circ} \mathrm{C}$ overnight with AHNAK antibody in the dilution of 1:50 (Santa Cruz Biotechnology) or primary Ki-67 antibody in the dilution of 1:50 (Dako, glostrup, Denmark, clone MiB-1). Then, the slides were incubated with secondary antibody and product visualization (Dako) was performed with diaminobenzidine substrate as the chromogen. Finally, the sections were counterstained with Mayer's hematoxylin and washed with distilled water and PBS each time. Ki-67 index was calculated as the percentage of immunoreactive nuclei per 200 cells in five high power fields. The low and high indices were considered as staining of $0-10 \%$ and $\geq 10 \%$ cell nuclei, respectively. The image analysis and total gray value were estimated by the GSM-2000P pathology image analysis system (Heima Zhuhai, China).

\section{Flow cytometric analysis measuring cell apoptosis}

After treatments with transfected AHNAKexpressing vector, an annexin V-FITC/PI (propidium iodide) apoptosis detection kit (BD PharMingen) was 
used to label the harvested U87/U251 glioma cells according to the instruction of manufacture. A BD FACSCalibur (USA) flow cytometer was used to quantify the apoptotic and necrotic cells. The cells of early apoptotic (Annexin V-positive and PI-negative) or late apoptotic (Annexin V-positive and PI-positive) phases were included in cell death determinations.

\section{Mouse xenograft model}

U87 and U251 cells stable transfect AHNAKvector or control vector were collected and suspended at a concentration of $1 \times 10^{7}$ cells $/ \mathrm{ml}$ in PBS, respectively. Then inoculated subcutaneously 200ul cancer cell suspension into dorsal flanks of (female, 4-6w) each nude mouse (five in each group) by using 1 -ml injector. Tumor size was measured every four days. After 28 days, the mice were killed and necropsies were performed. Tumor growth was monitored by caliper measurement once or twice every week for 28 days. Tumor volume was calculated as follows: $\mathrm{V}=\mathrm{L} \times 1^{2} \times 0.5$, where $\mathrm{L}$ and 1 represent the larger and the smaller tumor diameters, respectively. The animal experiments were performed in accordance with institutional guidelines.

\section{Statistical analysis}

Comparisons between groups were analyzed with $t$ tests and $\chi^{2}$ tests. Overall survival curves and relapse-free curves were plotted according to the Kaplan-Meier method, and the log-rank test was used for comparison. Survival was counted from the day of the surgery. All of the differences were statistically significant at the $\mathrm{P}<0.05$ level. The statistical analyses were performed using the SPSS16.0 software.

\section{Results}

\section{AHNAK expression is significantly down-regulated in glioma and correlated with the clinicopathological characteristics and prognosis of patients}

To analyze the expression of AHNAK in glioma, we tested AHNAK mRNA level in a panel of six human glioma cell lines by using the qRT-PCR assay. As Figure 1A showed, compared with the normal gliocyte line, the AHNAK mRNA levels are down-regulated in four human glioma cell lines (SHG-44, A172, U87, U251), especially in A172, U87 and $\mathrm{U} 251$ cell lines ( $\mathrm{P}<0.001$, respectively).

In order to confirm the results that we found in cell lines, we tested AHNAK mRNA level in 73 cases of glioma tissue samples and other 30 cases of normal brain tissues. As expected, the mRNA level of AHNAK significantly decreased in glioma issue samples $(\mathrm{P}<0.001)$ showed in Figure 1B. The representative immunohistochemical staining of
AHNAK in glioma tissues and corresponding normal brain tissues were showed in Figure 1D.

Further, we tested AHNAK mRNA expression level in 73 cases of glioma tissue samples, to explore whether it is associated with clinical pathological parameters. Depended on the average mRNA level of AHNAK, there were 44 patients with low AHNAK and 29 patients with high AHNAK. The results as shown in Table 1, AHNAK expression was observed to be negatively correlated with the glioma histopathology $(\mathrm{P}<0.001)$. But no significant correlations between AHNAK expression and other clinicopathologic factors, including the patient age $(\mathrm{P}=0.384)$ and gender $(\mathrm{P}=0.462)$. Meanwhile, in survival analyses, we found that low expression of AHNAK was a significant and independent prognostic factor for poor survival of glioma patients showed in Figure 1C.

\section{Overexpression of AHNAK could inhibit glioma cell line proliferation, invasion and induce apoptosis}

Then, in vitro studies, we elucidated the biological effects of AHNAK in glioma cell lines. The AHNAK-expressing vector or control vector was transfected into U87 and U251 cell lines, respectively. The efficiency of transfection was tested by western blotting (Figure 2A). Compared with the control group, the proliferation assay showed that the high ectopic expression of AHNAK in U87 and U251 cells could inhibit the cell proliferation dramatically (Figure 2B). In cell transwell assay, we found that overexpression AHNAK could attenuate U87 and U251 cells invasion capacity (Figure 2C). At the same time, we tested the degree of apoptosis in glioma cell lines induced by AHNAK. And we found that overexpression AHNAK could induce U87 and U251 cells to be apoptosis (Figure 2D). These results mentioned above indicate that AHNAK might play a tumor suppression role in glioma cell lines.

\section{Overexpression of AHNAK in glioma cell lines could inhibit tumor growth in vivo}

In vitro experiments and clinicopathological analysis results suggested that AHNAK played a tumor suppression role in glioma and it might be negatively correlate with the extent of tumor metastasis. To confirm the physiological relevance of our in vitro observations, we examined the effects of AHNAK on tumor growth with the xenograft model of human glioma cells in nude mice. Generally, U87 or U251 cells infected with AHNAK-expressing vector or control vector lentivirus were injected into the flank of nude mice. After 28 days, the mice were killed and tumors size was measured. We could observe 
compared with the control group, the mean of tumor sizes of AHNAK overexpression group exhibited a significant decrease (Figure 3). Furthermore, we examined the expression of $\mathrm{Ki}$-67-positive cells in these tumors. Obviously, a marked decrease in the number of Ki-67-positive cells was observed in the AHNAK overexpression group compared to the control group in both glioma cells as well as the previous results (Figure 4). These results suggested that AHNAK could repress glioma proliferation and metastasis in vivo.

\section{Discussion}

Glioma, the most common and intractable type of intracranial tumor in adults, is a clinically heterogeneous disease with an extremely poor prognosis because of their highly invasive growth pattern and their frequent resistance to therapies [27]. The clinical outcomes of glioma patients depend heavily on histopathological features [4]. The glioblastoma multiform (GBM), as the most malignant tumor type, is almost invariably fatal with an overall survival of just over one year only [4]. Therefore, over the past decades, significant developments have been made in profiling the molecular signatures of glioma using the most advanced cytobiology and molecular biological technique. To improve treatment efficacy, a better understanding of glioma pathogenesis at the genetic and molecular levels is urgently needed $[3$, 28].
A

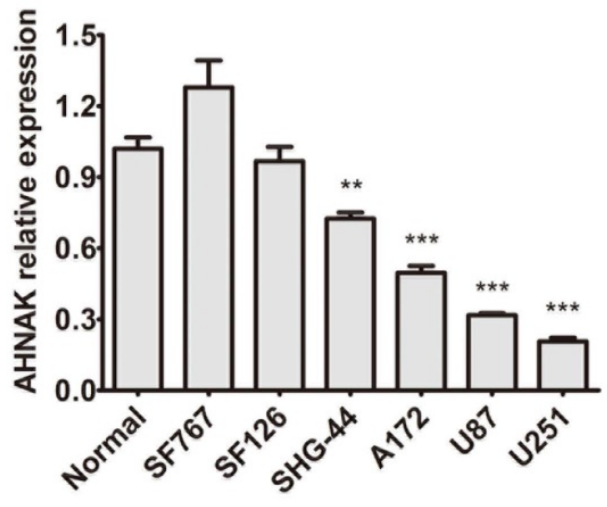

D
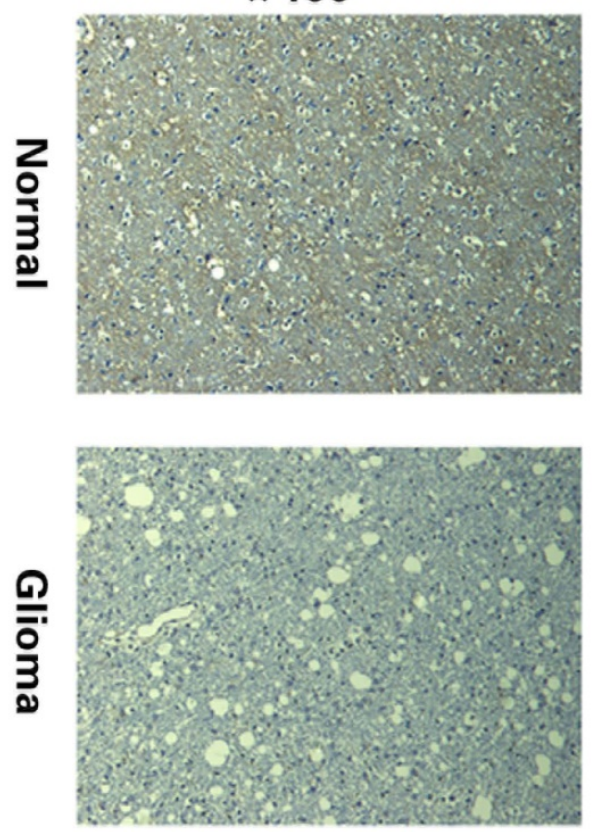

B

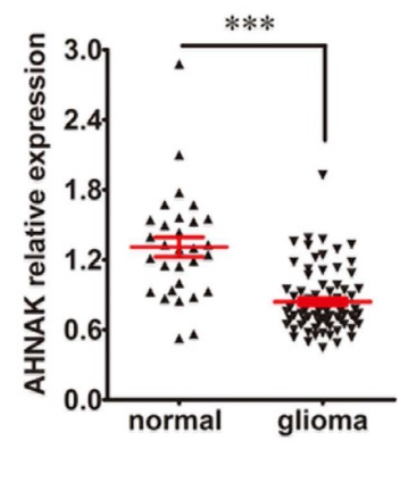

$\times 200$
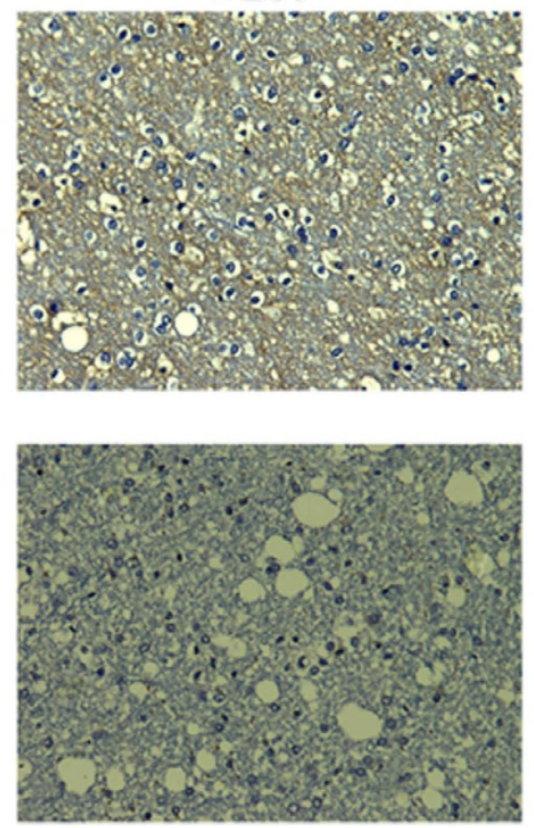

C

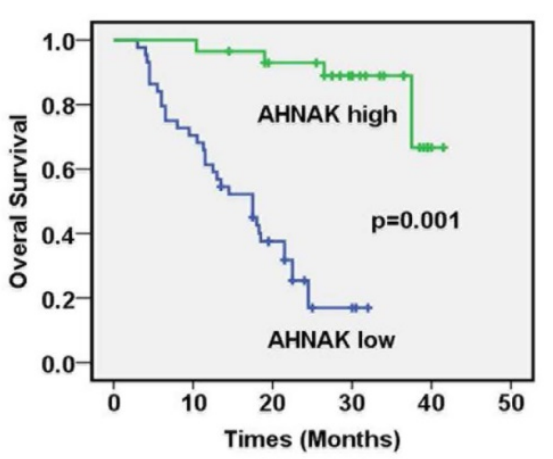

$\times 400$
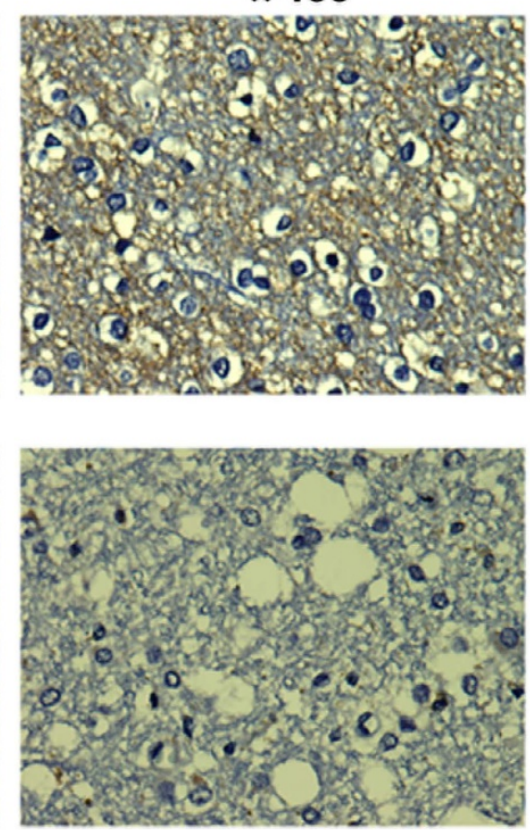

Figure 1. AHNAK expression is significantly down-regulated in glioma. (A) The AHNAK expression level analysed by qRT-PCR in six human glioma cell lines. Normal gliocyte line as control for normalization. The standard deviations (SD) is represent by the error bars, $* * P<0.01$ and $* * * P<0.001$. (B) Expression levels of AHNAK in 73 glioma tissues and the corresponding 30 cases of normal adjacent tissues, ***P $<0.001$. (C) Overall Survival (OS) curves for 73 patients of glioma with high or low AHNAK expression (The green line represents AHNAK high, the bule line represents AHNAK low, the green line with the vertical bar represents AHNAK high-censored and the bule line with the vertical bar represents AHNAK low-censored), P=0.001. (D) Representative immunohistochemistry images of AHNAK expression in normal brain tissue and glioma. Original magnification X100, X200 and X400. 
A

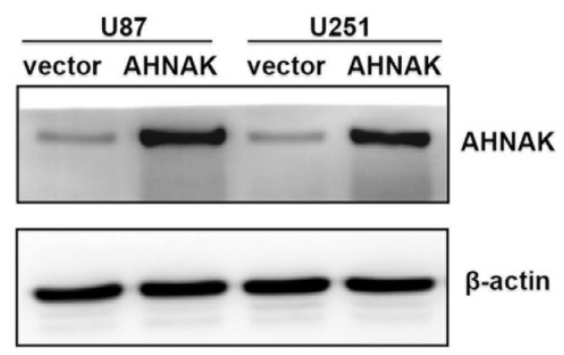

B

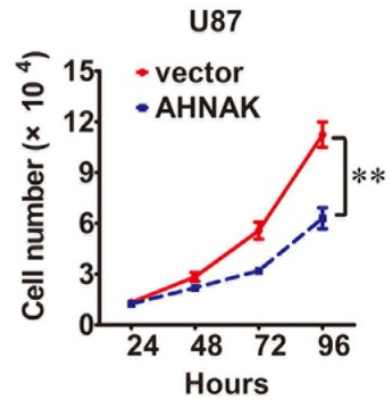

U251

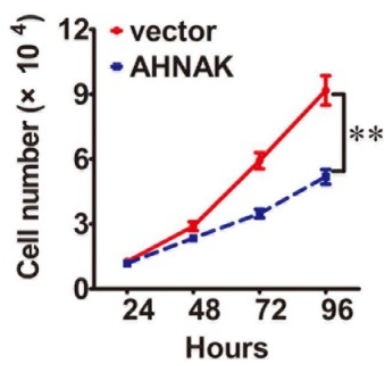

C

AHNAK
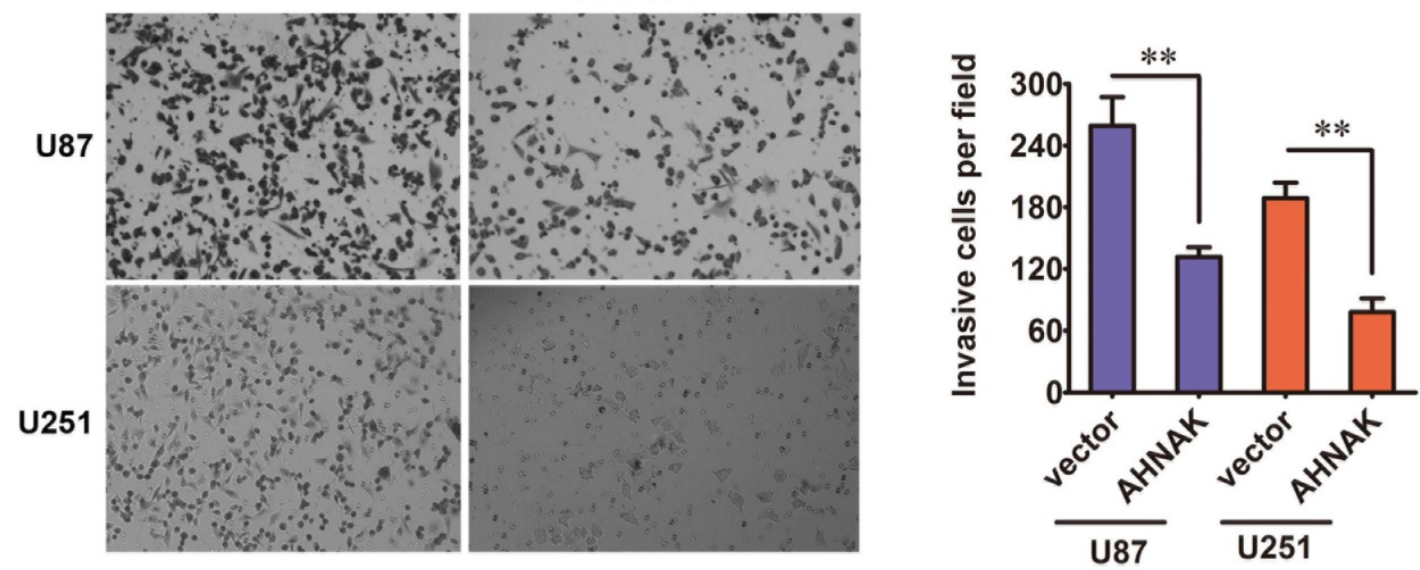

D
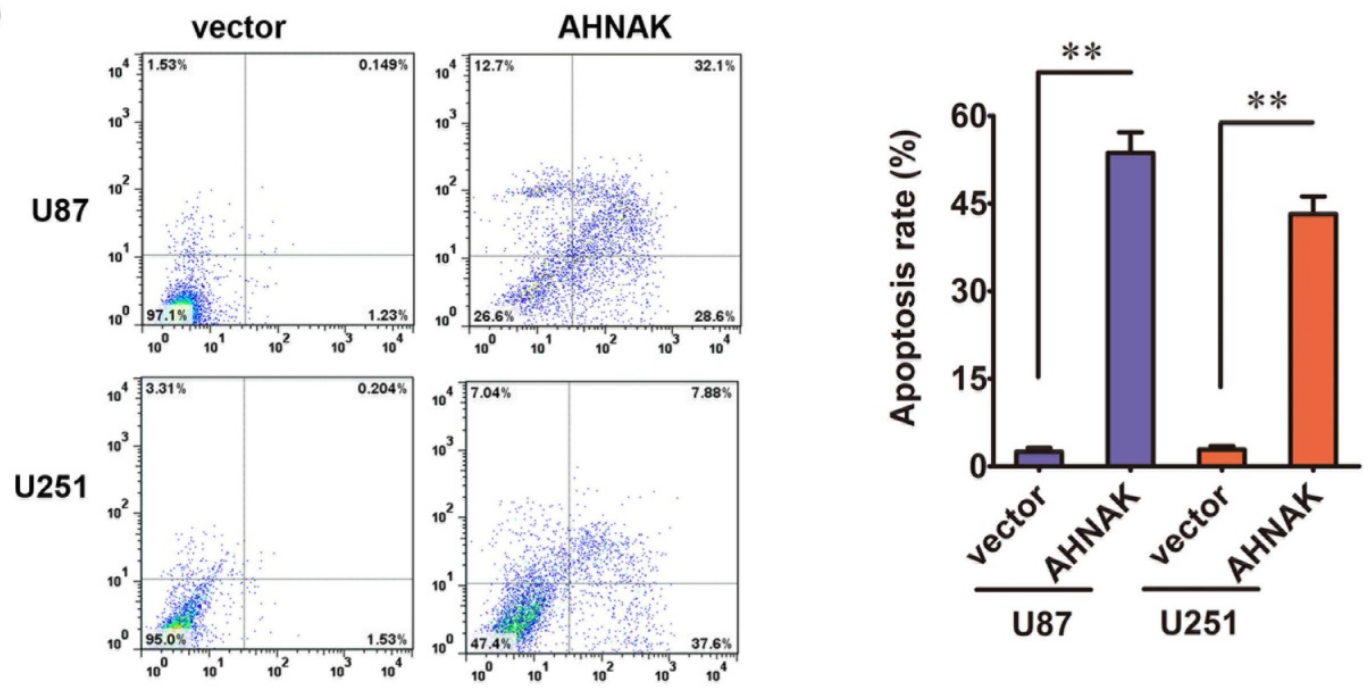

Figure 2. AHNAK could inhibit glioma cell line proliferation, invasion and induce apotosis. (A) The relative levels of AHNAK expression between AHNAK and vector in U87 and U251 cells by western blotting with the loading control of $\beta$-actin. (B) The growth of U87 and U251 cells infected with AHNAK-overexpression vector or control vector was assayed by MTT, **P $<0.01$. (C) The invasion assay was measured through transwell assays with matrigel, **P $<0.01$. (D) The effect of AHNAK or vector on U87 and U251 cells cell apoptosis, $* * \mathrm{P}<0.01$.

AHNAK is a large protein of approximately 700 $\mathrm{kDa}$ found at the inner plasma membrane [19] in different cell types which was originally identified in neuroblastoma cell lines and involves in lots of cellular processes and pathways [29]. And studies on the molecular function of AHNAK over the past years have been experiencing dramatic alterations, with the direction to tumor metastasis nowadays. A study showed that AHNAK in combination with macrophage migration inhibitory factor was a strong predictor of poor survival in the patient of laryngeal carcinoma [23]. Recently, AHNAK was found that it was significantly enriched in purified pseudopodia of six different metastatic cancer cell lines [16]. And further investigation revealed that the knockdown of AHNAK resulted in pseudopodial retraction, EMT reversal as well as impaired tumor cell migration and invasion [16]. Another research reported similar 
findings on AHNAK that AHNAK gene expression was shown to be significantly higher in seven different mesothelioma cell lines with regards to tumor cell migration and invasion [22]. Similarly, knockdown of AHNAK resulted in decreased migration and invasion in the mesothelioma cell lines [22]. Meanwhile, AHNAK enabled mammary carcinoma cells to produce extracellular vesicles that increase neighboring fibroblast cell motility [24]. On the contrary of the above-mentioned studies which AHNAK was considered as an oncogenic protein, it also has been demonstrated to play a role of a tumor suppressor. However, the function of AHNAK in glioma still remains largely undefined and relative research is rare.
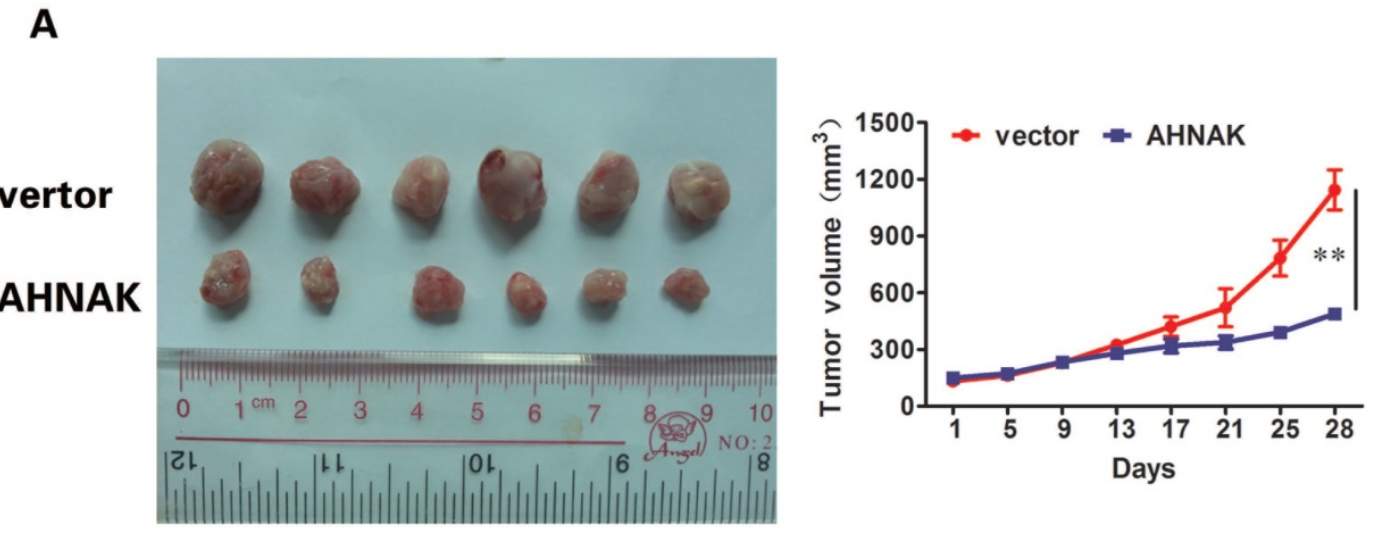

U87
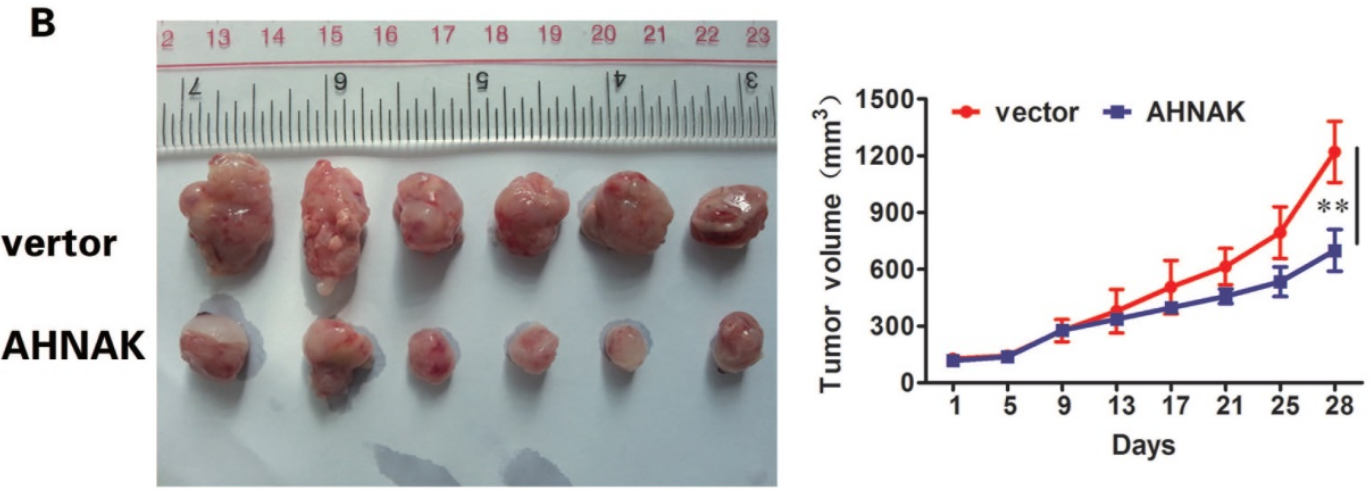

\section{U251}

Figure 3. AHNAK could inhibit glioma growth in vivo. U87 (A) and U251 (B) cells infected with AHNAK or vector lentivirus were injected into flank of nude mice. Tumor size taken from mouse xenograft models (left, $n=6$ per group). The tumor sizes of AHNAK overexpression group were smaller than control group. The growth curves of the xenograft tumors were plotted in the panel (right, $n=6$ per group). All results were expressed as the mean \pm SD of three independent experiments, $* * P<0.01$.
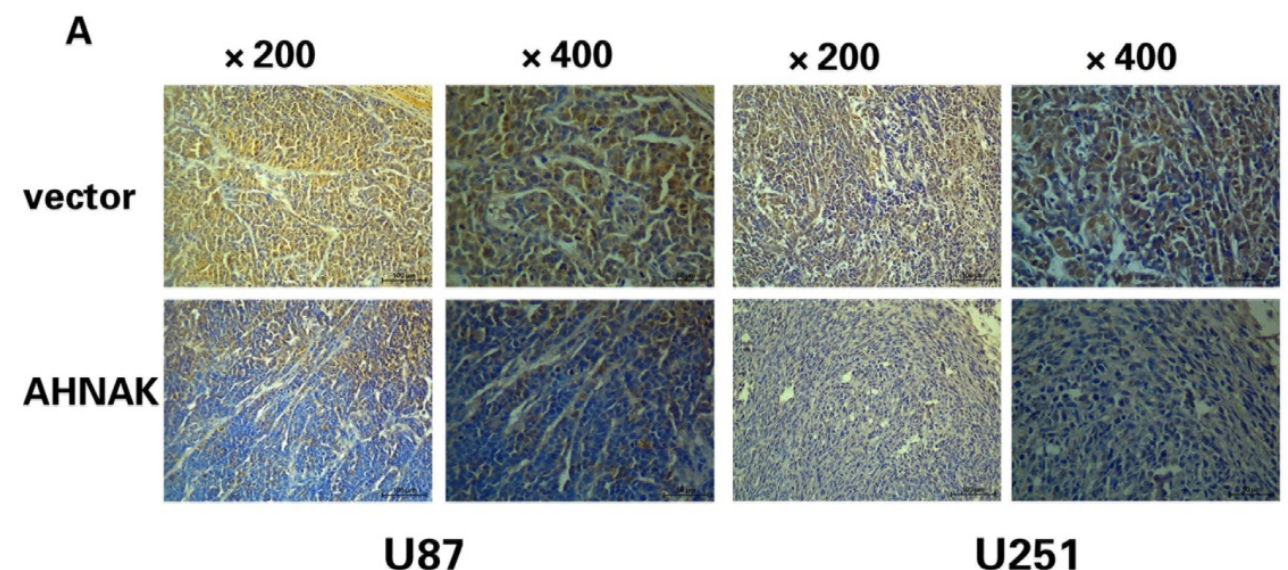

B

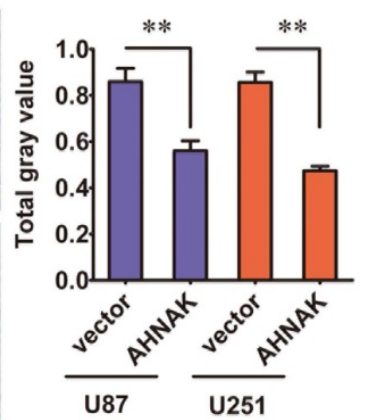

Figure 4. AHNAK could inhibit the expression of Ki-67 in xenograft tumors. (A) The Ki-67 stained sections derived from xenograft tumors in each group (left: U87, right: U251). Original magnification X200 and X400. (B) The gray value of Ki-76 expressed in xenograft tumors were summarized in the panel. All results were expressed as the mean \pm SD of three independent experiments, $* * P<0.01$. 
In this study, we demonstrated the biological function of AHNAK in glioma. We discovered that the expression of AHNAK was lower in four of six human glioma cell lines. In addition, we also found that the decreased expression of AHNAK was most markedly in the glioma patients and its expression was negatively correlated with the vital clinicopathological characteristic. Meanwhile, in survival analyses, we also knew the low expression of AHNAK was a significant and independent prognostic factor for poor survival of glioma patients. The same results were found in recent studies of melanoma [21] and triple-negative breast cancer [25] showing that AHNAK was down-regulated and considered as an independent prognostic factor related to the poor survival of patients. However, in the laryngeal carcinoma, tumoral AHNAK was overexpression significantly and associated with poor survival [23]. Taken together, the lower expression of AHNAK is possibly meaning the higher glioma histopathology and poorer overall survival but none of relevance with the age and gender. And no relevant reported previously. Therefore, we consider that AHNAK could be likely to become one of new and independent prognostic factor for glioma patient.

In vitro studies, the results indicated that overexpression of AHNAK could inhibit glioma cell proliferation, could weaken glioma cell invasion in transwell assay and even could induce glioma cell to be apoptosis in both of U87 and U251 cell lines. These results mentioned above indicated that AHNAK might play a tumor suppression role in glioma cell lines. Meanwhile, in vivo studies, as showed that infected with AHNAK vectors glioma cells could decrease tumor of xenograft model growth and weight. Considered Ki-67 is a marker of proliferation and a prognostic indicator [30], we examined of the expression of Ki-67-positive cells in the tumors, and Ki-67 low expression was found in overexpression AHNAK group which possibly prompted the ability of proliferation and aggressiveness was decreased in AHNAK overexpression group. In a meta-analysis of human gliomas, Ki-67 overexpression predicted poor progression-free survival and poor overall survival [31]. The Ki-67 expression tested in vivo study was in accord with the conclusion in histopatholgy and survival analyses mentioned above. Therefore, in both of vitro and vivo studies manifested the AHNAK could influence the proliferation, invasion, apoptosis and metastasis in glioma cell further verified the role of AHNAK playing in glioma.

With above of these results, we confirmed the function displayed by AHNAK in suppress tumor progression. These findings are consistent with reports of decreased AHNAK expression in and human breast cancer [12] and melanoma [21]. Lee et al. adequately reported the AHNAK played a vital role in the TGF- $\beta /$ Smad signaling pathway through a variety of experiments. AHNAK could promote TGF- $\beta$-induced transcriptional activity of R-Smad to stimulate Smad3 localization into nucleus leading to a negative regulation of cell growth. Moreover, overexpression of AHNAK could downregulate of c-Myc and cyclin D1/D2 resulted in cell cycle arrest and growth retardation [12]. In that case, the mechanism of action was shown to be through modulation of the TGF- $\beta /$ Smad signaling pathway. As we known, TGF- $\beta$ represents with controversial roles owns contrasting roles in cancer biology: one as tumor suppressor based on the ability to induce growth arrest and apoptosis and, on the hand, as a tumor promotor, based on the ability to induce angiogenesis and EMT [32]. Therefore, the role of AHNAK is most likely determined by TGF- $\beta$ and those regulating its activity in these tumors. Meanwhile, the specific or different stage in cancer progression could perhaps also be involved [29]. In addition, another recent research has shown that down-regulation of AHNAK can occur during the transformation of melanocytes into metastatic melanoma which may be required for the expression of functional cadherin- 1 and that it correlates with poor patient prognosis [21]. However, the specific mechanism of the down-regulation of AHNAK in glioma has not been elucidated. And this is also the limitation of our study. In vitro studies, we found the phenomenon of apoptosis induced by overexpression of AHNAK in glioma cell lines. Hence, we boldly propose our hypothesis that the mechanism of the down-regulation of AHNAK in glioma possibly involves in TGF- $\beta$-relative signaling pathway based on its ability to induce apoptosis mentioned above or in other apoptosis-related signaling pathways. And this is also the direction, in which we need to intensively study to understand the molecular biology of AHNAK in glioma.

In conclusion, our study demonstrated the apoptotic effects of AHNAK as the potential tumor suppression role in glioma, and the negative correlation of its expression level with the malignant status. Moreover, AHNAK could at least predict the prognosis for glioma patient. Taken together, even though more detailed tumor suppressive mechanisms for AHNAK in glioma need to be revealed, our findings provided a preclinical basis in developing AHNAK as a reliable clinical predictor of outcome for glioma patients and a new biomarker for treatment response and new target for therapy in glioma. 


\section{Abbreviations}

AHNAK: AHNAK nucleoprotein; WHO: World Health Organization; qRT-PCR: Quantitative Reverse Transcription-PCR; MTT: Methyl Thiazolyl Tetrazolium; DMEM: Dulbecco Modified Eagle Medium; OD: Optical Density; V-FITC/PI: VFluorescein Isothiocyanate /Propidium Iodide; GBM: Glioblastoma Multiform; EMT: EpithelialMesenchymal Transition; TGF- $\beta$ : Transforming Growth Factor- $\beta$.

\section{Acknowledgments}

This work was supported by funds from the National Key Technology Research and Development Program of the Ministry of Science and Technology of China (2014BAI04B02, Qing Liu).

\section{Competing Interests}

The authors have declared that no competing interest exists.

\section{References}

[1] Siegel R, Ma J, Zou Z, et al. Cancer statistics, 2014. CA Cancer J Clin. 2014; 64: 9-29.

[2] Jemal A, Bray F, Center MM, et al. Global cancer statistics. CA Cancer J Clin. 2011; 61: 69-90.

[3] Louis DN, Perry A, Reifenberger G, et al. The 2016 World Health Organization Classification of Tumors of the Central Nervous System: a summary. Acta Neuropathol. 2016; 131: 803-820.

[4] Wen PY and Kesari S. Malignant gliomas in adults. N Engl J Med. 2008; 359: 492-507.

[5] Omuro A and DeAngelis LM. Glioblastoma and other malignant gliomas: a clinical review. JAMA. 2013; 310: 1842-1850.

[6] Chen R, Smith-Cohn M, Cohen AL, et al. Glioma Subclassifications and Their Clinical Significance. Neurotherapeutics. 2017: 1-14.

[7] Ma KX, Wang HJ, Li XR, et al. Long noncoding RNA MALAT1 associates with the malignant status and poor prognosis in glioma. Tumour Biol. 2015; 36: 3355-3359.

[8] Wang P, Liu YH, Yao YL, et al. Long non-coding RNA CASC2 suppresses malignancy in human gliomas by miR-21. Cell Signal. 2015; 27: 275-282.

[9] Shtivelman E, Cohen FE and Bishop JM. A human gene (AHNAK) encoding an unusually large protein with a 1.2-microns polyionic rod structure. Proc Natl Acad Sci USA. 1992; 89: 5472-5476.

[10] Hashimoto T, Amagai M, Parry DA, et al. Desmoyokin, a 680 kDa keratinocyte plasma membrane-associated protein, is homologous to the protein encoded by human gene AHNAK. J Cell Sci. 1993; 105 (Pt 2): 275-286.

[11] Masunaga T, Shimizu H, Ishiko A, et al. Desmoyokin/AHNAK protein localizes to the non-desmosomal keratinocyte cell surface of human epidermis. J Invest Dermatol. 1995; 104: 941-945.

[12] Lee IH, Sohn M, Lim HI, et al. Ahnak functions as a tumor suppressor via modulation of TGF $\beta /$ Smad signaling pathway. Oncogene. 2014; 33: 4675-4684.

[13] Han WQ, Xia M, Xu M, et al. Lysosome fusion to the cell membrane is mediated by the dysferlin C2A domain in coronary arterial endothelial cells. J Cell Sci. 2012; 125: 1225-1234.

[14] Ozorowski G, Milton S and Luecke H. Structure of a C-terminal AHNAK peptide in a 1:2:2 complex with S100A10 and an acetylated $\mathrm{N}$-terminal peptide of annexin A2. Acta Crystallogr D Biol Crystallogr. 2013; 69: 92-104.

[15] Shin JH, Lee SH, Kim YN, et al. AHNAK deficiency promotes browning and lipolysis in mice via increased responsiveness to $\beta$-adrenergic signalling. Sci Rep. 2016; 6: 23426

[16] Shankar J, Messenberg A, Chan J, et al. Pseudopodial actin dynamics control epithelial-mesenchymal transition in metastatic cancer cells. Cancer Res. 2010; 70: 3780-3790.

[17] Haase H, Alvarez J, Petzhold D, et al. Ahnak is critical for cardiac Ca(V)1.2 calcium channel function and its beta-adrenergic regulation. FASEB J. 2005; 19: 1969-1977.

[18] Gentil BJ, Benaud C, Delphin C, et al. Specific AHNAK expression in brain endothelial cells with barrier properties. J Cell Physiol. 2005; 203: 362-371.

[19] Cardinali B, Cappella M, Provenzano C, et al. MicroRNA-222 regulates muscle alternative splicing through Rbm24 during differentiation of skeletal muscle cells. Cell Death Dis. 2016; 7: e2086.
[20] Lim HJ, Kim J, Park CH, et al. Regulation of c-Myc Expression by Ahnak Promotes Induced Pluripotent Stem Cell Generation. J Biol Chem. 2016; 291 : 752-761.

[21] Sheppard HM, Feisst V, Chen J, et al. AHNAK is downregulated in melanoma, predicts poor outcome, and may be required for the expression of functional cadherin-1. Melanoma Res. 2016; 26: 108-116.

[22] Sudo $H$, Tsuji AB, Sugyo A, et al. AHNAK is highly expressed and plays a key role in cell migration and invasion in mesothelioma. Int J Oncol. 2014; 44: 530-538.

[23] Dumitru CA, Bankfalvi A, Gu X, et al. AHNAK and inflammatory markers predict poor survival in laryngeal carcinoma. PLoS One. 2013; 8: e56420.

[24] Silva TA, Smuczek B, Valadão IC, et al. AHNAK enables mammary carcinoma cells to produce extracellular vesicles that increase neighboring fibroblast cell motility. Oncotarget. 2016; 7: 49998-50016.

[25] Chen B, Wang J, Dai D, et al. AHNAK suppresses tumour proliferation and invasion by targeting multiple pathways in triple-negative breast cancer. J Exp Clin Cancer Res. 2017; 36: 65.

[26] Bhargava S, Patil V, Mahalingam $\mathrm{K}$, et al. Elucidation of the genetic and epigenetic landscape alterations in RNA binding proteins in glioblastoma. Oncotarget. 2017; 8: 16650-16668.

[27] Cuddapah VA, Robel S, Watkins S, et al. A neurocentric perspective on glioma invasion. Nat Rev Neurosci. 2014; 15: 455-465.

[28] Chen J, McKay RM and Parada LF. Malignant glioma: lessons from genomics, mouse models, and stem cells. Cell. 2012; 149: 36-47.

[29] Davis TA, Loos B and Engelbrecht AM. AHNAK: the giant jack of all trades. Cell Signal. 2014; 26: 2683-2693.

[30] Fraser AR, Bacci B, le CMA, et al. Epidermal Growth Factor Receptor and Ki-67 Expression in Canine Gliomas. Vet Pathol. 2016; 53: 1131-1137.

[31] Chen WJ, He DS, Tang RX, et al. Ki-67 is a valuable prognostic factor in gliomas: evidence from a systematic review and meta-analysis. Asian Pac J Cancer Prev. 2015; 16: 411-420.

[32] Cantelli G, Crosas-Molist E, Georgouli M, et al. TGFB-induced transcription in cancer. Semin Cancer Biol. 2017; 42: 60-69. 